Wolfgang E. Nagel • Dietmar B. Kröner • Michael M. Resch

Editors

\title{
High Performance Computing \\ in Science and \\ Engineering '11
}

Transactions of the High Performance

Computing Center, Stuttgart (HLRS) 2011

色 Springer 


\section{Contents}

Physics

P. Nielaba

The Influence of the Mass Ratio on Particle Acceleration

by the Filamentation Instability $\ldots \ldots \ldots \ldots \ldots \ldots \ldots \ldots \ldots \ldots \ldots$

P. Kilian, T. Burkart, and F. Spanier

The SuperN-Project: Neutrino Hydrodynamics Simulations

of Core-Collapse Supernovae . . . . . . . . . . . . . . . . 15

B. Müller, L. Hüdepohl, A. Marek, F. Hanke, and H.-Th. Janka

Simulation of Pre-planetesimal Collisions with Smoothed Particle

Hydrodynamics . . . . . . . . . . . . . . . . . . . . . . . . . . . . 29

R.J. Geretshauser, R. Speith, and W. Kley

Copper Substrate Catalyzes Tetraazaperopyrene Polymerization

W.G. Schmidt, E. Rauls, U. Gerstmann, S. Sanna, M. Landmann,

M. Rohrmüller, A. Riefer, S. Wippermann, and S. Blankenburg

QCD Critical Surfaces at Real and Imaginary $\mu$

O. Philipsen and Ph. de Forcrand

Higgs Boson Mass Bounds from a Chirally Invariant Lattice

Higgs-Yukawa Model

P. Gerhold, K. Jansen, and J. Kallarackal

Massive and Massless Four-Loop Integrals

P. Baikov, K. Chetyrkin, J.H. Kühn, P. Marquard, and M. Steinhauser

Solid State Physics

H. Fehske

Laser Ablation of Aluminium: Drops and Voids

J. Roth, J. Karlin, C. Ulrich, H.-R. Trebin, and S. Sonntag 
Cysteine on Gold: An ab-initio Investigation

B. Höffling, F. Ortmann, K. Hannewald, and F. Bechstedt

Ab-initio Calculations of the Vibrational Properties of Nanostructures

G. Bester and P. Han

Entropy and Metal-Insulator Transition in Atomic-Scale Wires:

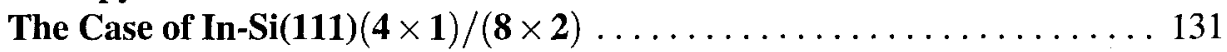

W.G. Schmidt, E. Rauls, U. Gerstmann, S. Sanna, M. Landmann,

M. Rohrmüller, A. Riefer, and S. Wippermann

Obtaining the Full Counting Statistics of Correlated Nanostructures

from Time Dependent Simulations

P. Schmitteckert

Phase Diagram of the $1 \mathrm{D}$ t-J Model

A. Moreno, A. Muramatsu, and S. Manmana

Chemistry

C. van Wüllen

Constrained Density Functional Theory of Molecular Dimers

J.-H. Franke, N.N. Nair, L. Chi, and H. Fuchs

Atomistic Simulations of Electrolyte Solutions and Hydrogels

with Explicit Solvent Models

J. Walter, S. Deublein, S. Reiser, M. Horsch, J. Vrabec, and H. Hasse

cuVASP: A GPU-Accelerated Plane-Wave Electronic-Structure

Code

S. Maintz, B. Eck, and R. Dronskowski

Reacting Flows

D. Kröner

Assessment of Conventional Droplet Evaporation Models for Spray

Flames

M.R.G. Zoby, A. Kronenburg, S. Navarro-Martinez, and A.J. Marquis

Analysis of the Effects of Wall Boundary Conditions and Detailed Kinetics on the Simulation of a Gas Turbine Model Combustor Under

Very Lean Conditions

F. Rebosio, A. Widenhorn, B. Noll, and M. Aigner

Oxy-coal Combustion Modeling at Semi-industrial Scale

M. Müller, U. Schnell, and G. Scheffknecht 
Delayed Detached Eddy Simulations of Compressible Turbulent Mixing Layer and Detailed Performance Analysis of Scientific In-House Code TASCOM3D

M. Kindler, P. Gerlinger, and M. Aigner

Computational Fluid Dynamics

S. Wagner

Discontinuous Galerkin for High Performance Computational Fluid Dynamics (hpedg)

C. Altmann, A. Beck, A. Birkefeld, F. Hindenlang, M. Staudenmaier,

G. Gassner, and C.-D. Munz

Highly Efficient and Scalable Software for the Simulation of Turbulent

Flows in Complex Geometries . . . . . . . . . . . . . . . . . . . 289

D.F. Harlacher, S. Roller, F. Hindenlang, C.-D. Munz, T. Kraus, M. Fischer,

K. Geurts, M. Meinke, T. Klühspies, V. Metsch, and K. Benkert

A Computation Technique for Rigid Particle Flows in an Eulerian Framework Using the Multiphase DNS Code FS3D . . . . . . . . . . . . . 309

P. Rauschenberger, J. Schlottke, and B. Weigand

Optimization of Chaotic Micromixers Using Finite Time Lyapunov Exponents

A. Sarkar, A. Narváez, and J. Harting

Numerical Simulation of Particle-Laden Turbulent Flows Using LES . . . 337

M. Breuer and M. Alletto

Large-Eddy Simulation of Supersonic Film Cooling at Finite Pressure

Gradients

M. Konopka, M. Meinke, and W. Schröder

Prediction of Stability Limits of Combustion Chambers with LES

B. Pritz, F. Magagnato, and M. Gabi

Numerical Simulation of Laminar-Turbulent Transition on a Dolphin

Using the $\gamma$ - $\operatorname{Re}_{\theta}$ Model

D. Riedeberger and U. Rist

Wall Effects and Corner Separations for Subsonic and Transonic Flow

Regimes

A. Klein, S. Illi, K. Nübler, T. Lutz, and E. Krämer

Numerical Simulation of Helicopter Wake Evolution, Performance

and Trim

F. Bensing, M. Embacher, M. Hollands, B. Kutz, M. Keßler, and E. Krämer 
Parameter Study for Scramjet Intake Concerning Wall Temperatures

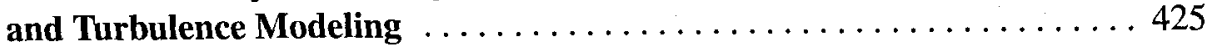
B. Reinartz

Unsteady Numerical Study of Wet Steam Flow in a Low Pressure Steam Turbine .

J. Starzmann, M.V. Casey, and J.F. Mayer

Turbulence Modelling for CFD-Methods for Containment Flows

A. Zirkel and E. Laurien

Transport and Climate

C. Kottmeier

The Transport of Mineral Dust Towards Hurricane Helene (2006)

J. Schwendike, S. Jones, H. Vogel, and B. Vogel

Numerical Modelling of Mediterranean Cyclones

C.-J. Lenz, U. Corsmeier, and C. Kottmeier

Modelling Near Future Regional Climate Change for Germany and Africa

H.-J. Panitz, P. Berg, G. Schädler, and G. Fosser

High-Resolution Climate Predictions and Short-Range Forecasts to Improve the Process Understanding and the Representation of Land-Surface Interactions in the WRF Model in Southwest Germany

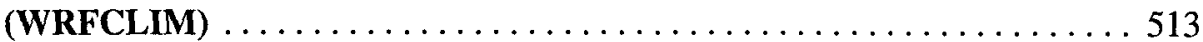
H.-S. Bauer, K. Warrach-Sagi, V. Wulfmeyer, T. Schwitalla, and M. Kirn

Direct Numerical Simulation and Implicit Large Eddy Simulation of Stratified Turbulence

S. Remmler and S. Hickel

Miscellaneous Topics

W. Schröder

Allocation of Economic Capital in Banking: A Simulation Approach 541 H.-P. Burghof and J. Müller

The Influence of Partial Melt on Mantle Convection

A.-C. Plesa and T. Spohn

Molecular Modeling of Hydrogen Bonding Fluids: Phase Behavior of Industrial Fluids

S. Eckelsbach, M. Bernreuther, C. Engin, G. Guevara-Carrion, Y.-L. Huang,

T. Merker, H. Hasse, and J. Vrabec 
"Brute-Force" Solution of Large-Scale Systems of Equations in a MPI-PBLAS-ScaLAPACK Environment

M. Roth, O. Baur, and W. Keller

Metallic Foam Structures, Dendrites and Implementation Optimizations for Phase-Field Modeling . . . . . . . . . . . . . . . . . . . . . . . . . 595

A. Vondrous, B. Nestler, A. August, E. Wesner, A. Choudhury, and J. Hötzer

Quaero 2010 Speech-to-Text Evaluation Systems . . . . . . . . . . . 607

S. Stüker, K. Kilgour, and F. Kraft

Accurate Simulation of Wireless Vehicular Networks Based on Ray

Tracing and Physical Layer Simulation

T. Gaugel, L. Reichardt, J. Mittag, T. Zwick, and H. Hartenstein

Reduction of Numerical Sensitivities in Crash Simulations

on HPC-Computers (HPC-10)

O. Mangold, R. Prohl, A. Tkachuk, and V. Trickov

Three-Dimensional Gyrotron Simulation Using a High-Order Particle-in-Cell Method

A. Stock, J. Neudorfer, B. Steinbusch, T. Stindl, R. Schneider, S. Roller,

C.-D. Munz, and M. Auweter-Kurtz 